02

\title{
Влияние примеси меди на электронную структуру и оптические свойства соединений $\mathrm{TmNi}_{5}$
}

\author{
() Ю.В. Князев ${ }^{1}$, А.В. Лукоянов ${ }^{1,2}$, Ю.И. Кузьмин ${ }^{1}$, А.Г. Кучин ${ }^{1}$, М. Vasundhara ${ }^{3}$ \\ ${ }^{1}$ Институт фризики металлов им. М.Н. Михеева Уральского отделения РАН, \\ 620990 Екатеринбург, Россия \\ ${ }^{2}$ Уральский фредеральный университет им. Б.Н. Ельцина, \\ 620002 Екатеринбург, Россия \\ ${ }^{3}$ CSIR-National Institute for Interdisciplinary Science and Technology, \\ Trivandrum 695019 , Kerala, India \\ e-mail: knyazev@imp.uran.ru
}

Поступила в редакцию 16.01.2018г.

Проведены исследования электронной структуры и оптических свойств соединений $\mathrm{TmNi}_{5-x} \mathrm{Cu}_{x}$ $(x=0,1,2,3)$. В приближении локальной электронной спиновой плотности с поправкой на сильные электронные взаимодействия в рамках метода LSDA $+U$ выполнены самосогласованные расчеты зонного спектра. Рассчитаны полные и парциальные плотности электронных состояний, связанные с атомами тулия, никеля и меди. В широком интервале длин волн $0.22-16 \mu \mathrm{m}$ методом эллипсометрии измерена оптическая проводимость, поведение которой интерпретируется для каждого соединения с учетом проведенных расчетов. Определены концентрационные зависимости плазменных и релаксационных частот электронов проводимости.

DOI: $10.21883 /$ OS.2018.06.46076.12-18

\section{Введение}

Интерес к исследованию интерметаллических соединений $\mathrm{RNi}_{5}$ ( $\mathrm{R}$ - редкоземельный металл) обусловлен уникальными особенностями их физико-химических свойств, открывающими большие возможности для функционального применения. На базе таких сплавов, отличающихся многообразием магнитных и электронных характеристик [1-6], разрабатываются материалы для постоянных магнитов, устройств с использованием магнитострикционных, магниторезистивных и магнетокалорических эффектов. Высокая способность к абсорбции и хранению атомарного водорода, обнаруженная в интерметаллидах указанного типа, способствует созданию энергоемких портативных аккумуляторов [7,8]. В процессе изучения таких соединений было установлено, что их транспортные и магнитные свойства существенно трансформируются при замещении никеля атомами других переходных $d$ - или $p$-металлов вследствие изменения параметров электронной структуры, кристаллического поля и обменных взаимодействий. Например, в ряде псевдобинарных соединений $\mathrm{RNi}_{5-x} \mathrm{M}_{x}$ обнаружены аномалии в поведении концентрационных зависимостей структурных, термодинамических и электрических параметров, магнитной восприимчивости, температуры магнитных переходов и т.д. [3,9-12]. Такие модификации физических свойств при внедрении примеси способствуют улучшению функциональных характеристик данных материалов, в частности существенно влияют на их абсорбционную способность.

Представителем указанной серии интерметаллидов является $\mathrm{TmNi}_{5}$, ряд физических свойств которого изу- чался в работах $[13,14]$. Данное бинарное соединение является ферромагнетиком с температурой Кюри $T_{\mathrm{C}}=4.5 \mathrm{~K}$ и выделяется в серии $\mathrm{RNi}_{5}$ тем, что обладает большим значением кристаллического поля, приводящим к сильной магнетокристаллической анизотропии [15]. При изучении свойств тройных сплавов $\mathrm{TmNi}_{5-x} \mathrm{Cu}_{x}$ [3], представляющих собой твердые растворы со структурой исходного соединения, было установлено, что с увеличением примеси меди наблюдается заметное изменение параметров кристаллической решетки, электронных и магнитных характеристик. Дополнительную информацию, необходимую для объяснения особенностей эволюции электронных и магнитных свойств тройных соединений на базе $\mathrm{TmNi}_{5}$ при замещении никеля атомами меди, могут представить совместные исследования их энергетических спектров и спектральных свойств. С этой целью в настоящей работе были проведены расчеты плотности электронных состояний интерметаллидов $\mathrm{TmNi}_{5-x} \mathrm{Cu}_{x}$ при $x=0,1,2$ и 3, на основе которых проведена интерпретация экспериментальных оптических данных.

\section{Расчет электронной структуры}

Соединения $\mathrm{TmNi}_{5-x} \mathrm{Cu}_{x} \quad(x=0,1,2,3)$ кристаллизуются в гексагональной структуре типа $\mathrm{CaCu}_{5}$ (пространственная группа симметрии $P 6 / \mathrm{mmm}$ ). Элементарная ячейка содержит одну формульную единицу c атомом $\mathrm{Tm}$ в кристаллографической позиции $1 \mathrm{a}$ $(0,0,0)$, два атома никеля располагаются в позициях типа $2 c(1 / 3,2 / 3,0)$ и три атома никеля - в позициях типа $3 g(1 / 2,0,1 / 2)$. В расчетах использо- 
вались экспериментальные величины параметров кристаллической решетки, определенные в работе [3]. Расчеты электронной структуры были выполнены в рамках метода LSDA $+U$ [16] в пакете программ Quantum ESPRESSO [17] с использованием обменнокорреляционного потенциала в приближении обобщенной градиентной поправки GGA версии PBE [18]. Волновые функции раскладывались по плоским волнам, взаимодействия между ионами и валентными электронами учитывались в рамках метода присоединенных плоских волн (PAW). В расчетах были использованы PAWпотенциалы Tm.GGA-PBE-paw-vl.0.UPF [19], Ni.pbe-nkjpaw_psl.0.1.UPF и Cu.pbe-dn-kjpaw_psl.0.2.UPF. Для получения достаточной сходимости в цикле самосогласования при расчете использовался энергетический предел для плоских волн, равный $60 \mathrm{Ry.} \mathrm{B} \mathrm{обратном}$ пространстве интегрирование производилось по сетке из $8 \times 8 \times 8$ k-точек. Для учета сильных электронных корреляций $4 f$-электронов Тm была включена поправка для параметров прямого кулоновского $(U=6.2 \mathrm{eV})$ и обменного (хундовского, $J=0.7 \mathrm{eV}$ ) взаимодействий. Данные величины близки к значениям, используемым ранее [20] и оцененным из экспериментальных данных [21]. В расчетах были получены величины спиновой поляризации $4 f$-состояний иона Тm, соответствующие магнитному моменту $2 \mu_{\mathrm{B}}$ на редкоземельный ион. Ионы никеля и меди во всех расчетах получены полностью немагнитными. Примесь замещения меди помещалась как в позиции типа $2 c$, так и $3 g$. Были проведены расчеты для всех возможных вариантов размещений, далее полученные самосогласованные плотности электронных состояний усреднялись с соответствующими весами.

Полные плотности электронных состояний $N(E)$ coединений $\mathrm{TmNi}_{5-x} \mathrm{Cu}_{x}(x=0,1,2,3)$ для двух направлений спина $\uparrow$ и $\downarrow$ представлены на рис. 1. Здесь же приводятся парциальные плотности для $4 f$-электронов Тm и $3 d$-электронов $\mathrm{Ni}$ и $\mathrm{Cu}$, дающие основной вклад в полную $N(E)$. Темные области, характеризуемые острыми максимумами, соответствуют парциальным плотностям $4 f$-электронов $\mathrm{Tm}$, находящихся в свободных и заполненных состояниях. В $\uparrow$-системе зон интенсивные пики, связанные с локализованными $f$-электронами, расположены в интервале $6.5-8.5 \mathrm{eV}$ ниже уровня Ферми $E_{\mathrm{F}}$. В зоне с противоположным направлением спина максимумы, связанные с этими электронами, находятся при энергиях $3.5-4.5 \mathrm{eV}$ ниже $E_{\mathrm{F}}$ и при $0.5-1.5 \mathrm{eV}$ выше $E_{\mathrm{F}}$. Заполненные электронные состояния до энергии $\sim-5 \mathrm{eV}$ связаны главным образом с $3 d$-зонами $\mathrm{Ni}$ и $\mathrm{Cu}$, формирующими энергетические полосы, которые по своей форме почти идентичны для обеих спиновых проекций. Серые области, которые расположены в валентной зоне, соответствуют $3 d$-состояниям $\mathrm{Cu}$. Увеличение содержания меди приводит к росту интенсивности и протяженности данной структуры, практически не влияя на положение ее центра тяжести. Отметим, что рассчитанные в данных соединениях энергии локализации примесной $3 d$-зоны $\mathrm{Cu}$ с максимумом в интервале 3-4 eV близки по величине к значениям, полученным

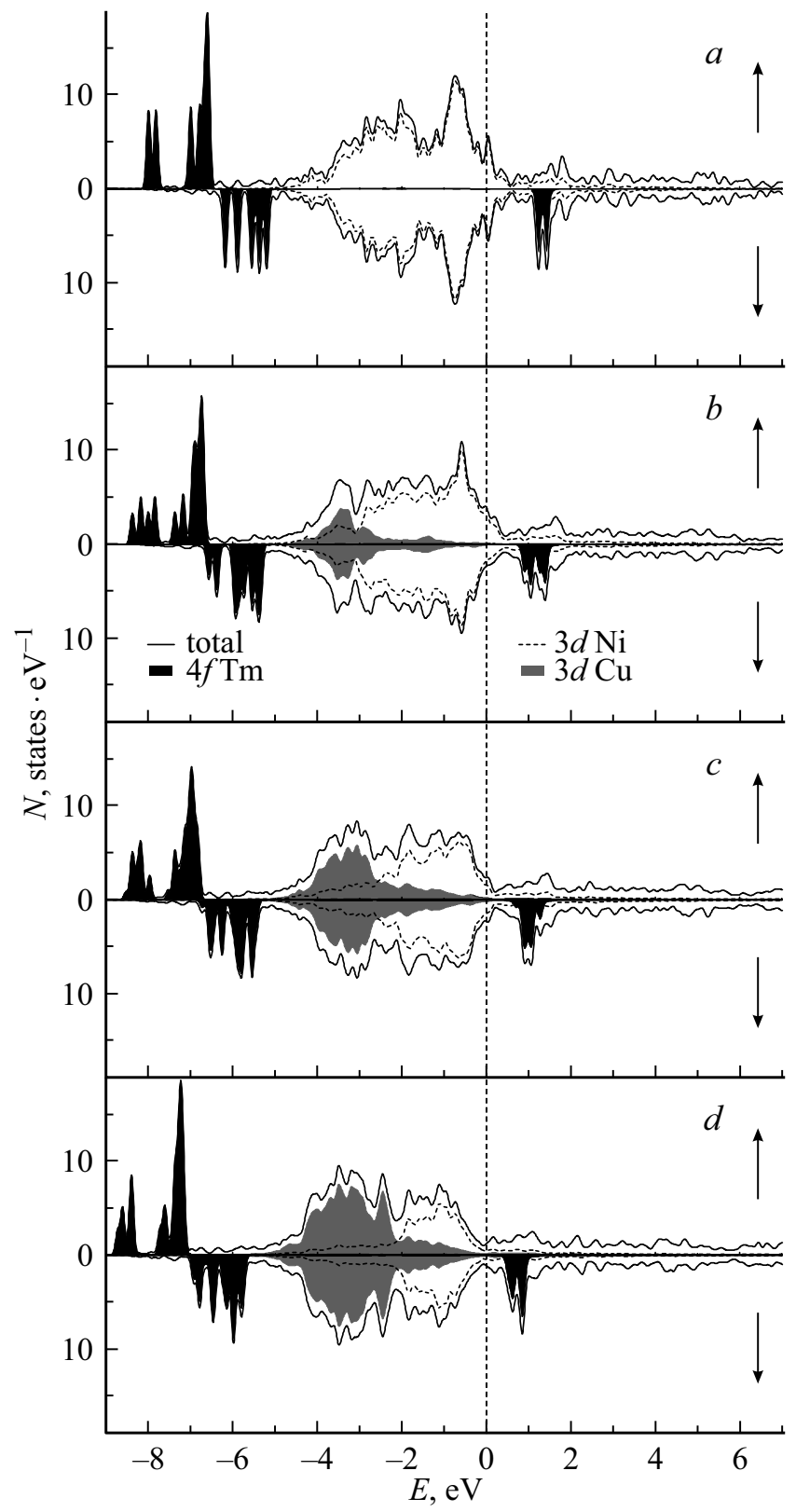

Рис. 1. Плотности электронных состояний соединений $\mathrm{TmNi}_{5}(a), \mathrm{TmNi}_{4} \mathrm{Cu}(b), \mathrm{TmNi}_{3} \mathrm{Cu}_{2}(c), \mathrm{TmNi}_{2} \mathrm{Cu}_{3}(d)$ для двух спиновых направлений. Представлены полные и парциальные плотности для $4 f$-зон Tm, $3 d$-зон Сu и $3 d$-зон Ni. Уровень Ферми соответствует нулю на шкале энергий.

для других интерметаллидов подобного типа, где никель замещается атомами меди [22,23].

\section{Результаты и обсуждение}

Соединения $\operatorname{TmNi}_{5-x} \mathrm{Cu}_{x}(x=0,1,2,3)$ были выплавлены в индукционной печи в тигле из оксида алюминия в атмосфере чистого аргона. Полученные образцы гомогенизировались в течение $8 \mathrm{~h}$ при температуpe $\sim 1100^{\circ} \mathrm{C}$. Результаты рентгеноструктурного анализа 


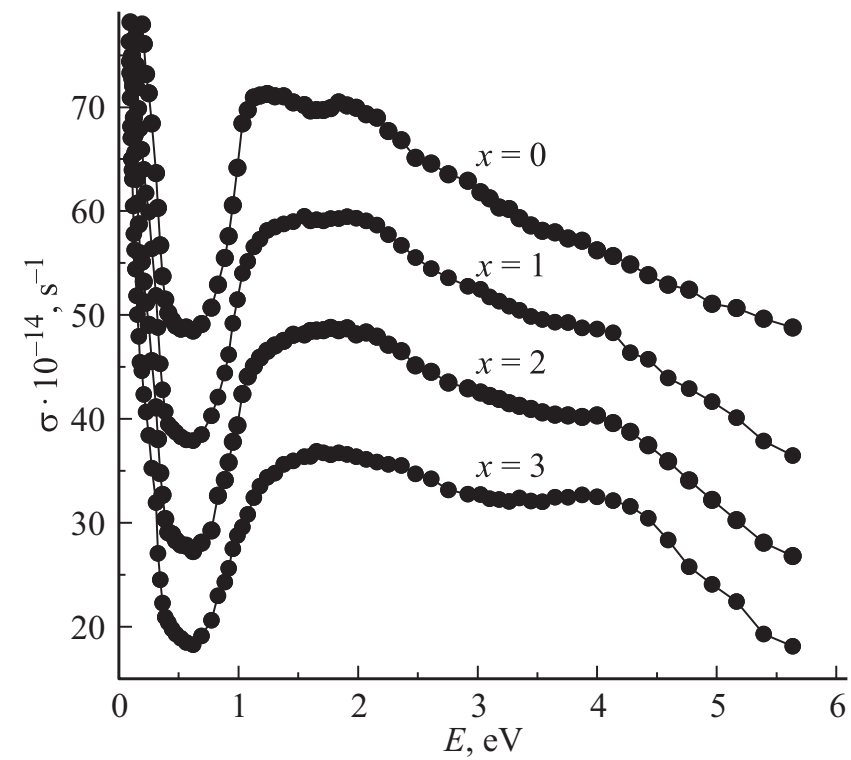

Рис. 2. Дисперсия оптической проводимости соединений $\mathrm{TmNi}_{5-x} \mathrm{Cu}_{x}(x=0,1,2,3)$. Кривые сдвинуты вверх по оси ординат относительно друг друга на 10 единиц.

показали, что все сплавы были однородными, однофазными и имели кристаллическую структуру типа $\mathrm{CaCu}_{5}$. Спектральные свойства соединений изучались при комнатной температуре в области длин волн $0.22-16 \mu \mathrm{m}$ $(E=0.078-5.64 \mathrm{eV})$. Оптические постоянные - показатели преломления $n$ и поглощения $k-$ измерены эллипсометрическим методом с вращающимся анализатором при углах падения света $70-80^{\circ}$ с погрешностью $1-3 \%$. Зеркальные поверхности образцов 14-го класса чистоты приготовлены механическим полированием на алмазных пастах. По значениям $n$ и $k$ рассчитаны плазменные и релаксационные частоты электронов проводимости, а также оптическая проводимость $\sigma=n k \omega / 2 \pi \omega(\omega-$ частота света) - величина, характеризующая интенсивность и частотную зависимость оптического отклика отражающей среды. В отличие от статической проводимости этот параметр чувствителен к изменению плотности электронных состояний не только в области уровня Ферми, но и во всем исследуемом спектральном интервале.

Экспериментальные спектры оптической проводимости данных соединений представлены на рис. 2 (кривые сдвинуты по оси ординат относительно друг друга на 10 единиц). Частотная дисперсия всех зависимостей $\sigma(E)$ в целом типична для металлоподобных сред, и ее структура показывает наличие вкладов от двух типов поглощения света - внутризонного (друдевского) и межзонного. В низкоэнергетической области при $E \leq \sim 0.5 \mathrm{eV}$ резкий рост $\sigma(E)$ с уменьшением частоты связан с ускорительным механизмом взаимодействия электронов с электромагнитной волной $\left(\sigma \sim \omega^{-2}\right)$, а влияние межзонного поглощения на оптические характеристики минимально. Численные значения оптических постоянных, измеренные в этом диапазоне, позволяют по классическим друдевским соотношениям определить релаксационные $\gamma$ и плазменные $\omega_{p}$ частоты электронов проводимости. Рассчитанная таким образом частота релаксации, аддитивно учитывающая все типы рассеяния электронов при их возбуждении электромагнитной волной (электрон-электронное, электрон-фононное, на дефектах и примесях), заметно возрастает при увеличении числа атомов замещения и принимает значения $2.8 \cdot 10^{14}$ $\left(\mathrm{TmNi}_{5}\right), 3.1 \cdot 10^{14}\left(\mathrm{TmNi}_{4} \mathrm{Cu}\right), 3.3 \cdot 10^{14}\left(\mathrm{TmNi}_{3} \mathrm{Cu}_{2}\right)$ и $3.6 \cdot 10^{14} \mathrm{~s}^{-1}\left(\mathrm{TmNi}_{2} \mathrm{Cu}_{3}\right)$. Плазменная частота $\omega_{p}$, характеризующая коллективные осцилляции электронов проводимости, с ростом концентрации примеси проявляет тенденцию к уменьшению: $6.5 \cdot 10^{15}\left(\mathrm{TmNi}_{5}\right)$, $6.2 \cdot 10^{15}\left(\mathrm{TmNi}_{4} \mathrm{Cu}\right), 5.9 \cdot 10^{15}\left(\mathrm{TmNi}_{3} \mathrm{Cu}_{2}\right), 5.7 \cdot 10^{15} \mathrm{~s}^{-1}$ $\left(\mathrm{TmNi}_{2} \mathrm{Cu}_{3}\right)$. Данный параметр, зависящий от строения электронного спектра и эффектов межэлектронного взаимодействия, пропорционален плотности состояний на уровне Ферми [24]. Учитывая этот фактор, следует отметить, что уменьшение величины $\omega_{p}$ с ростом содержания примеси $\mathrm{Cu}$ прямым образом коррелирует с результатами расчета электронной структуры, в которых величины $N\left(E_{\mathrm{F}}\right)$ также показывают существенное снижение (рис. 1). С использованием значений плазменных и релаксационных частот для каждого соединения рассчитывались друдевские вклады в оптическую проводимость, которые приведены на рис. 3.

Немонотонный характер зависимостей $\sigma(E)$ свидетельствует о преобладающей роли квантовых электронных переходов. Спектры оптической проводимости всех четырех соединений в этом интервале характеризуются интенсивной полосой межзонного поглощения, профиль которой существенно зависит от состава материала. Если в структуре соответствующей кривой бинарного $\mathrm{TmNi}_{5}$ проявляются два отчетливо выраженных максимума (при 1.2 и $1.8 \mathrm{eV}$ ), то по мере замещения никеля атомами меди эти две особенности постепенно преобразуются в единый широкий максимум. Кроме того, с ростом количества примеси в зависимости $\sigma(E)$ возникает новая протяженная полоса поглощения с максимумом вблизи $4 \mathrm{eV}$, не проявляемая в спектре бинарного сплава.

Наблюдаемые на рис. 2 изменения спектрального профиля частотных зависимостей оптической проводимости в процессе увеличения содержания $\mathrm{Cu}$ связаны с происходящей модификацией электронной структуры данных соединений в пределах нескольких электронвольт от уровня Ферми. В связи с этим имеет смысл сопоставить экспериментальные зависимости $\sigma(E)$ в области межзонного поглощения света с соответствующими характеристиками, рассчитанными из плотностей электронных состояний (представлены на рис. 3). Здесь же для всех соединений приведены частотные зависимости межзонных оптических проводимостей, полученные вычитанием друдевских вкладов из экспериментальных спектров. Для расчета дисперсии межзонного вклада в оптическую проводимость на базе электронной структуры по схеме, предложенной в [25], выполнена 


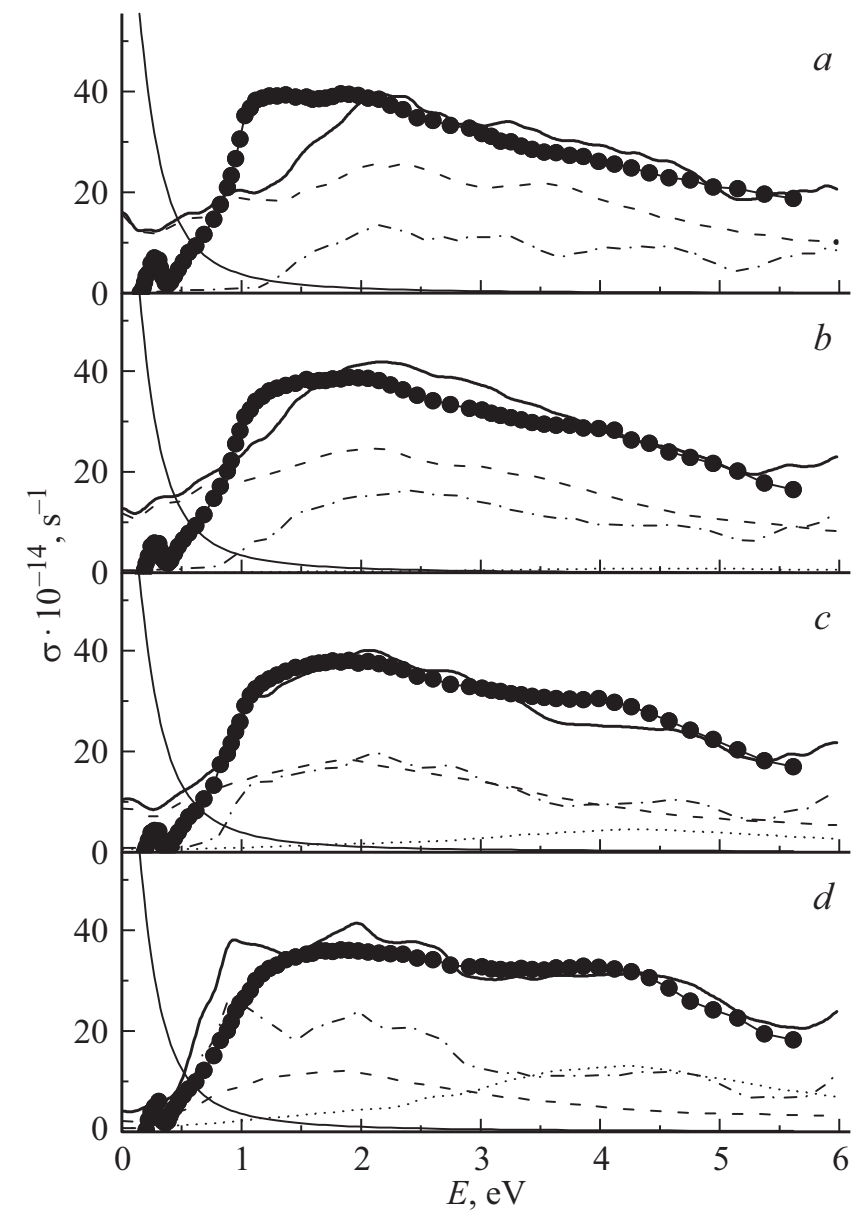

Pис. 3. Спектры межзонной оптической проводимости соединений $\mathrm{TmNi}_{5}(a), \mathrm{TmNi}_{4} \mathrm{Cu}(b), \mathrm{TmNi}_{3} \mathrm{Cu}_{2}(c), \mathrm{TmNi}_{2} \mathrm{Cu}_{3}(d)$. Точки - эксперимент, толстые сплошные кривые - расчет из полных плотностей состояний. Штриховые, штрихпунктирные и пунктирные линии - парциальные вклады межзонных переходов $d$-p-типа в атомах $\mathrm{Ni}, f-d$-типа в атомах $\mathrm{Tm}$ и $d-p$-типа в атомах $\mathrm{Cu}$. Тонкие сплошные линии - друдевский вклад.

свертка парциальных плотностей состояний на каждом неэквивалентном ионе элементарной ячейки с учетом правил отбора по квантовым числам $n^{\prime}=n \pm 1$. Затем все полученные результаты были просуммированы в соответствии с количеством ионов данного типа в элементарной ячейке. Оказалось, что наиболее существенный вклад в конечную величину $\sigma(E)$ бинарного $\mathrm{TmNi}_{5}$ дают свертки по $d$-p-состояниям $\mathrm{Ni}$ и $d-f$ состояниям $\mathrm{Tm}$. При этом $d-p$-переходы на ионах $\mathrm{Ni}$ существенны во всем энергетическом интервале, а $d-f$ переходы на ионах Tm дают сопоставимый вклад только при энергиях выше $\sim 1 \mathrm{eV}$. Данные вклады ответственны за образование широких полос межзонного поглощения, спектральный профиль которых плавно модифицируется с уменьшением содержания атомов никеля. В тройных соединениях $\mathrm{TmNi}_{5-x} \mathrm{Cu}_{x}$ с $x=1,2$ и 3 к указанным выше вкладам добавляются свертки $p-d$-состояний $\mathrm{Cu}$. Соответствующие интегральные величины вкладов для $p-d$-состояний $\mathrm{Cu}$ формируют полосу, интенсивность которой в интервале $3-5 \mathrm{eV}$ показывает значительный рост при увеличении уровня легирования. На рис. 3 для всех соединений представлена также сумма всех сверток по ионам элементарной ячейки.

Сравнение показывает, что в целом дисперсия теоретических кривых межзонной оптической проводимости качественно воспроизводит основные особенности соответствующих зависимостей, наблюдаемых в опытных спектрах. В соответствии с вычислениями для всех соединений предсказывается существование интенсивной полосы поглощения, формируемой квантовыми переходами между состояниями, разделяемыми уровнем Ферми. Расчет также подтвердил, что структура этой полосы трансформируется в зависимости от содержания примеси $\mathrm{Cu}$, а характер локализации примесной $3 d$ зоны $\mathrm{Cu}$, предсказанный вычислениями, соответствует эксперименту. Поскольку в расчете не учитывались матричные элементы межзонных переходов, то сравнение с экспериментом является качественным и в деталях тонкой структуры расчетных и опытных кривых межзонных $\sigma(E)$ нет полной корреляции. Например, в случае бинарного сплава $\mathrm{TmNi}_{5}$ существенное несоответствие в форме частотных зависимостей теоретической и экспериментальной оптической проводимости проявляется в интервале энергий $1-2 \mathrm{eV}$.

\section{Заключение}

В рамках самосогласованных расчетов, проведенных методом LSDA $+U$ с учетом сильных корреляционных поправок в $4 f$-оболочке тулия, определена электронная структура интерметаллических соединений $\mathrm{TmNi}_{5-x} \mathrm{Cu}_{x}(x=0,1,2,3)$. Установлены основные особенности трансформации спектров плотности электронных состояний и оптической проводимости, происходящие при замещении никеля атомами меди. Идентифицирована природа электронных состояний, формирующих полосы межзонного поглощения света. На основе полученных плотностей состояний рассчитаны межзонные оптические проводимости, поведение которых сравнивается с экспериментом. Показано, что характер частотной дисперсии экспериментальных зависимостей $\sigma(E)$ в области квантового поглощения света удовлетворительно описывается в рамках проведенных расчетов. Из оптических постоянных, измеренных в друдевской области спектра, для всех соединений определены плазменные и релаксационные частоты электронов проводимости.

Работа выполнена в рамках государственного задания ФАНО России (тема „Электрон“, № 01201463326) при частичной поддержке РФФИ (проекты 16-52-48012, 17-52-45056). А.В. Лукоянов благодарит за поддержку Правительство Российской Федерации (постановление № 211 контракт № 02.A03.21.0006). 


\section{Список литературы}

[1] Joshi D.A., Tomy C.V., Rana D.S., Nagarajan R., Malik S.K. // Solid State Commun. 2006. V. 137. P. 225. doi 10.1016/j.ssc.2005.11.005

[2] Kumar P., Suresh K.G., Nigam A.K., Gutfleisch O. // J. Phys. D: Appl. Phys. 2008. V. 41. N 24. P. 245006. doi 10.1088/0022-3727/41/24/245006

[3] Kuchin A.G., Ermolenko A.S., Kulikov Yu.A., Khrabrov V.I., Rosenfeld E.V., Makarova G.M., Lapina T.P., Belozerov Ye.V. // J. Magn. Magn. Mater. 2006. V. 303. N 1. P. 119. doi 10.1016/j.jmmm.2005.10.235

[4] Wang X., Chen R., Zhang Y., Chen C., Wang Q. // Mater. Lett. 2007. V. 61. N 4-5. P. 1101. doi 10.1016/j.matlet.2006.06.056

[5] Rocco D.L., Amaral J.S., Leitão J.V., Amaral V.S., Reis M.S., Fernandes R.P., Pereira A.M., Araújo J.P., Martins N.V., Tavares P.B., Coelho A.A. // Phys. Rev. B. 2009. V. 79. P. 014428. doi 10.1103/PhysRevB.79.014428

[6] Yao J., Isnard O., Morozkin A.V., Ivanova T.I., Koshkid'ko Yu.S., Bogdanov A.E., Nikitin S.A., Suski W. // J. Solid State Chem. 2015. V. 222. P. 123. doi 10.1016/j.jssc.2014.11.016

[7] Sakintuna B., Lamari-Darkrim F., Hirscher M. // Int. J. Hydrogen Energy. 2007. V. 32. N 9. P. 1121. doi 10.1016/j.ijhydene.2006.11.022

[8] Zhao X., Ma L. // Int. J. Hydrogen Energy. 2009. V. 34. P. 4788. doi 10.1016/j.ijhydene.2009.03.023

[9] Kuchin A.G., Ermolenko A.S., Khrabrov V.I., Makarova G.M., Belozerov E.V. // J. Magn. Magn. Mater. 1996. V. 159. P. L309. doi 10.1016/0304-8853(96)00322-8

[10] Lizárraga R., Bergman A., Björkman T., Liu H.-P., Andersson Y., Gustafsson T., Kuchin A.G., Ermolenko A.S., Nordström L., Eriksson O. // Phys. Rev. B. 2006. V. 74. P. 094419. doi 10.1103/PhysRevB.74.094419

[11] Haldar A., Dhiman I., Das A., Suresh K.G., Nigam A.K. // J. Alloys Compd. 2011. V. 509. N 9. P. 3760. doi 10.1016/j.jallcom.2011.01.012

[12] Toliński T. // Mod. Phys. Lett. B. 2007. V. 21. N 8. P. 431. doi 10.1142/S0217984907013079

[13] Gubbens P.C.M., van der Kraan A.M., Buschow K.H.J. // J. Magn. Magn. Mater. 1985. V. 50. N 2. P. 199. doi 10.1016/0304-8853(85)90182-9

[14] Barthem V.M.T.S., Gignoux D., Schmitt D., Creuzet G. // J. Magn. Magn. Mater. 1989. V. 78. P. 56. doi 10.1016/03048853(89)90086-3

[15] Kayzel F.E., Franse J.J.M., Colpa J.H.P., Kim-Ngan N.-H., Tai L.T., Radwański R.J., Gignoux D. // J. Magn. Magn. Mater. 1996. V. 157-158. P. 409. doi 10.1016/0304-8853(95)01278-8

[16] Anisimov V.I., Aryasetiawan F., Lichtenstein A.I. // J. Phys.: Condens. Matter. 1997. V. 9. N 4. P. 767. doi 10.1088/0953-8984/9/4/002

[17] Giannozzi P., Baroni S., Bonini N., Calandra M., Car R., Cavazzoni C., Ceresoli D., Chiarotti G.L., Cococcioni M., Dabo I., Dal Corso A., de Gironcoli S., Fabris S., Fratesi G., Gebauer R., Gerstmann U., Gougoussis C., Kokalj A., Lazzeri M., Martin-Samos L., Marzari N., Mauri F., Mazzarello R., Paolini S., Pasquarello A., Paulatto L., Sbraccia C., Scandolo S., Sclauzero G., Seitsonen A.P., Smogunov A., Umari P., Wentzcovitch R.M. // J. Phys.: Condens. Matter. 2009. V. 21. P. 395502. doi 10.1088/0953-8984/21/39/395502
[18] Perdew J.P., Burke K., Ernzerhof M. // Phys. Rev. Lett. 1996. V. 77. N 18. P. 3865. doi 10.1103/PhysRevLett.77.3865

[19] Topsakal M., Wentzcovitch R.M. // Comp. Mat. Sci. 2014. V. 95. P. 263. doi 10.1016/j.commatsci.2014.07.030

[20] Князев Ю.В., Лукоянов А.В., Кузьмин Ю.И., Gupta S., Suresh K.G. // ФТT. 2015. T. 57. № 12. C. 2289; Knyazev Yu.V., Lukoyanov A.V., Kuz'min Yu.I., Gupta S., Suresh K.G. // Phys. Solid State. 2015. V. 57. N 12. P. 2357. doi 10.1134/S1063783415120185

[21] Gupta S., Suresh K.G., Nigam A.K., Lukoyanov A.V. // J. Alloys Compd. 2015. V. 640. P. 56. doi 10.1016/j.jallcom.2015.02.126

[22] Князев Ю.В., Лукоянов А.В., Кузьмин Ю.И., Кучин А.Г. // ФНT. 2015. Т. 41. № 12. C. 1313; Knyazev Yu.V., Lukoyanov A.V., Kuz'min Yu.I., Kuchin A.G. // Low Tempr. Phys. 2015. V. 41. N 12. P. 1313. doi $10.1063 / 1.4935696$

[23] Князев Ю.В., Лукоянов А.В., Кузьмин Ю.И., Кучин А.Г. // ФTT. 2015. T. 57. № 5. C. 853; Knyazev Yu.V., Lukoyanov A.V., Kuz'min Yu.I., Kuchin A.G. // Phys. Solid State. 2015. V. 57. N 5. P. 866 doi $10.1134 / \mathrm{S} 1063783415050145$

[24] Каганов М.И., Слезов В.В. // ЖЭТФ. 1957. Т. 32. № 6. C. 1496; Kaganov M.I., Selezov V.V. // JETP 1957. V. 5. N 6. P. 1216.

[25] Некрасов И.А., Князев Ю.В., Кузьмин Ю.И., Кучин А.Г., Анисимов В.И. // ФММ. 2004. Т. 97. № 2. С. 13; Nekrasov I.A., Knyazev Yu.V., Kuz'min Yu.I., Kuchin A.G., Anisimov V.I. // Phys. Met. Metallography. 2004. V. 97. N 2. P. 129. 